\title{
REVIEW \\ Ethnicity and rehabilitation outcomes: the Needs Assessment Checklist
}

\author{
P Kennedy ${ }^{1,2}$, A Kilvert ${ }^{2}$ and L Hasson ${ }^{2}$
}

Study design: Retrospective cohort study with matched samples.

Objectives: To investigate whether significant differences in rehabilitation outcomes exist between different ethnic groups, using the Needs Assessment Checklist (NAC).

Settings: Tertiary care, spinal cord injury rehabilitation unit (National Spinal Injuries Centre), Stoke Mandeville Hospital, UK.

Methods: Rehabilitation outcomes and demographic information were obtained from the NAC. Data on 461 individuals were included in the study. Analysis of variance was employed to investigate differences in rehabilitation outcomes between various ethnic groups, across both the whole cohort and matched samples.

Results: Significant differences were evident across the different rehabilitation domains when ethnicity was examined, in particular within the domains of physical healthcare and psychological well-being. Within the unmatched data set, significant differences were found to exist in 3 of the 10 rehabilitation domains, and with the matched data set within 4 of the 10 domains. The results indicated that the cohort as a whole made significant improvements from the first to the second NAC within all rehabilitation domains.

Conclusion: Results indicate that ethnicity may have an impact on rehabilitation outcomes for individuals with spinal cord injury. Further investigation is needed to explore the nature of this relationship, and the future role of targeted interventions focusing on improving rehabilitation outcomes within the domains of physical and psychological care, in particular for individuals from different ethnic backgrounds.

Spinal Cord (2015) 53, 334-339; doi:10.1038/sc.2015.14; published online 17 February 2015

\section{INTRODUCTION}

All patients should have the same consistent access to high-quality rehabilitation services, irrespective of their ethnic origin, and as a consequence have the same opportunities to achieve the greatest possible functional outcomes. Much of the variation in health outcomes across different illnesses occurs as a consequence of ethnicity. ${ }^{1}$ In addition, routine data collection is poor, particularly in relation to follow-up and data analysis. ${ }^{2,3}$

In the UK there is little information available on the prevalence of spinal cord injury (SCI) in people from Black and minority ethnic backgrounds (BME). Furthermore, the literature on BME and rehabilitation outcomes within SCI worldwide is sparse and mixed in terms of what areas are found to differ. Research in this field has been primarily conducted in the USA, and this presents validity issues when considered within a European context because of pronounced differences in healthcare structures. In addition, within Europe the term BME is prevalent, whereas in studies conducted in the USA authors typically use 'ethnic minority' to a greater extent. The term BME will be used where possible in this article to provide clarity and continuity.

There is much disagreement as to whether there is a legitimate association between ethnicity disparities in treatment access and quality of care, ${ }^{4,5}$ or whether these differences can instead be accounted for by mediating factors such as level of household income and education. ${ }^{6}$ Within the current literature, differences have been found across multiple domains, including BME patients having a higher likelihood of secondary complications, psychological distress, pain, poorer wheelchair quality and other vulnerabilities relating to assisted technology, geographic location and socioeconomics. ${ }^{7,8}$

\section{REHABILITATION OUTCOMES}

Disparities in rehabilitation outcomes are well documented. Lad et al. ${ }^{9}$ explored the relationship between ethnicity and markers such as length of stay and in-hospital complications. Injury characteristics (level of injury and trauma type) and other patient covariates including age and gender were controlled for. African-American and Hispanic populations were found to have significantly longer hospital stays compared with Caucasians and Asians. African Americans and American Indians were found to be significantly more likely to have higher complications rates when compared with other ethnic groups. Furthermore, African-American and Asian individuals were found to have a lower likelihood of being discharged to an acute rehabilitation programme. This study clearly presents significant disparities between people from White and BME backgrounds, although these findings are somewhat limited given the self-report nature of the study.

Within physical rehabilitation outcomes, the influence of ethnicity has been explored previously using hierarchical regression analyses to control for variables in acute and rehabilitation care outcomes. ${ }^{10,11}$ No

${ }^{1}$ Oxford Institute of Clinical Psychology Training, University of Oxford, Oxford, UK and ${ }^{2}$ Department of Clinical Psychology, The National Spinal Injuries Centre, Stoke, Mandeville Hospital, Buckinghamshire, UK

Correspondence: Professor P Kennedy, Department of Clinical Psychology, The National Spinal Injuries Centre, Stoke, Mandeville Hospital, Mandeville Road, Aylesbury, Buckinghamshire HP21 8AL, UK.

E-mail: paul.kennedy@hmc.ox.ac.uk

Received 29 September 2014; revised 6 January 2015; accepted 9 January 2015; published online 17 February 2015 
differences were found between White and BME groups across a variety of outcomes, including functional independence at discharge, change during rehabilitation, length of stay and hospitalisation length. Furthermore, no significant differences were found in long-term outcomes when post-rehabilitation medical complications, number of non-routine clinic visits, overall self-reported handicap and hospitalisation days were measured. However, the BME group had a higher likelihood of having pressure ulcers compared with White individuals. This finding was replicated in another study that conducted interviews within three model rehabilitation hospitals in the USA. ${ }^{12}$ A differing prevalence of pressure ulcers according to ethnicity was evident; African-American and American-Indian individuals had a higher incidence compared with their Caucasian and Hispanic counterparts. Being of a particular ethnicity was found to be a risk factor for having a current pressure ulcer and one within the last year, despite many variables such as age, injury severity and social support being controlled for. A higher level of social support was significantly associated with a reduced risk for pressure ulcers.

Functional outcomes have been found to be significantly different within the domains of self-care and mobility when individuals from a non-Hispanic White, non-Hispanic Black, and Hispanic background were studied. ${ }^{13}$ At discharge, paraplegic and tetraplegic individuals had significantly poorer gains in self-care and mobility if they were from a non-Hispanic Black ethnic background compared with those who were non-Hispanic White or Hispanic. At 1-year follow-up, these differences in self-care and mobility ceased to exist. These results indicate that non-Hispanic Black individuals were unable to make as many gains within two critical aspects of spinal cord rehabilitation, and as such, at discharge, had less independence relative to the other two ethnic groups. Overall, these studies provide mixed support regarding whether there is a discrepancy in physical rehabilitation outcomes on account of ethnicity within SCI.

When looking at more widely defined physical outcomes, Krause et $a .^{6}$ found there to be significant differences between ethnicities when using a cross-sectional sample of survey data. Results indicated a significantly higher amount of poor health days and hospitalisation in African-American individuals. However, on two of three health outcomes where disparities were found, these effects were found to be mediated by education and household income; that is to say, the underlying factors of education and household income go some way to explicating the observed relationship between ethnicity and rehabilitation outcomes (see Figure 1). Mediating variables were found to account for a significantly higher amount of variance in outcomes than ethnicity or gender, indicating their pivotal role in studies on ethnicity.

\section{PSYCHOLOGICAL REHABILITATION OUTCOMES}

Several studies have reported significant differences in psychological rehabilitation outcomes across various ethnicities. One study that examined the effect of ethnicity in a sample of over 500 individuals

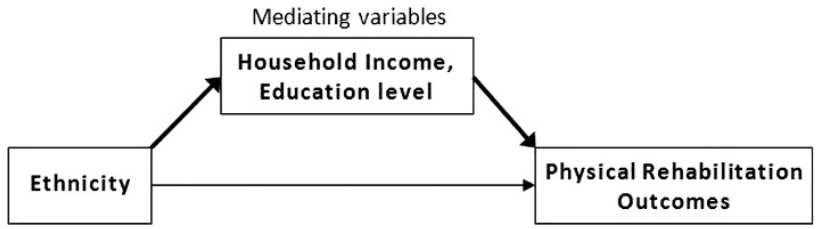

Figure 1 The observed relationship between ethnicity and rehabilitation outcomes is mediated by underlying variables such as household income and education level. found significant differences in subjective well-being. ${ }^{3}$ Specifically, white individuals reported higher well-being related to specific life areas, as opposed to more global outcomes such as health, in particular within finances and employment opportunities. Many of these differences were reported by the authors to be in areas where those from a BME background have been traditionally disadvantaged. Arango-Lasprilla et al. ${ }^{14}$ found further evidence of psychological wellbeing varying on account of ethnicity. The authors retrospectively examined life satisfaction across over 3000 individuals using the Satisfaction with Life scale at 1 year post injury. They found African Americans to have poorer scores compared with both Asians and Caucasians after controlling for marital status, cause of injury, employment, functional independence at discharge and stay length in acute care.

Similar results have been found in the context of a 6-year longitudinal study with differences evident in subjective well-being (favouring Caucasian individuals) and participation but not in actual health outcomes. ${ }^{15}$

\section{MODELS OF EXPLANATION}

Separating the effect of an individual's socioeconomic status and that of their ethnicity has been highlighted as an area of great difficulty. It has been hypothesised that being from a BME background acts more so as a proxy than as a predictor variable in explaining outcome after SCI. ${ }^{16}$ Two large case-control studies were conducted using Spinal Cord Injury Model System data, where different ethnic groups were matched according to age, gender, level of education, occupation, level of injury, American Spinal Injury Association (ASIA) Impairment Scale grade, aetiology, care sponsor and geographic region. Acute, rehabilitation and long-term outcomes were examined. White and African-American individuals were found not to differ significantly on any of the acute and rehabilitation outcome measures, including the functional independence measure, length of stay in hospital, cost, place of residence after discharge and outcomes related to medical complications. By controlling for demographic, medical and geographical characteristics, ethnicity was found to be largely unrelated to long-term outcomes; no differences were evident in medical complications, self-reported life satisfaction, level of handicap and physical or mental health. One significant difference was found in relation to mobility, with non-Whites reporting significantly greater handicap. On the whole, differences were accounted for by either sociodemographic or aetiological differences between ethnic groups. Overall, Putze et al. ${ }^{16}$ concluded that the variations across different outcome measures previously reported in the literature could not be attributed to ethnic background. In previous research, these variables were not as extensively matched for, which may be indicative of why significant differences were found. The authors therefore suggest that ethnicity acts as a proxy for other variables such as injury severity, occupation or level of education, which in themselves may be associated with poor outcomes after SCI, a finding that has been replicated in other research. ${ }^{17}$

\section{CURRENT RESEARCH}

The current literature does, to some extent, support the notion that discrepancies may exist in rehabilitation outcomes as a result of ethnicity, with a pattern of more prominent differences found in markers of psychological well-being and within physical health outcomes, particularly in relation to pressure ulcers. The relationship between ethnicity and physical/psychological outcomes within SCI has been demonstrated to be a complex area in which further investigation is needed. ${ }^{16,17}$ In addition, data collection within ethnicity research has 
historically been quite poor, an issue that has hindered previously conducted reviews and contributed to a limited current understanding of the relationship between ethnicity and SCI. Recent improvements in recording ethnicity information will mean that new studies can ascertain whether the disparities previously reported are valid. Such research will be instrumental in driving the evolution of spinal cord rehabilitation within Europe.

This study will explore differences in rehabilitation outcomes in SCI between three ethnic groups, using a sample of people with newly acquired SCI who were completing an inpatient multi-disciplinary rehabilitation programme at a Spinal Injuries Centre in the UK.

\section{MATERIALS AND METHODS}

\section{Participants}

Study One. This study utilised data that were routinely collected from all individuals admitted to The National Spinal Injuries Centre, Stoke Mandeville Hospital, UK, between 2008 and 2012. Six hundred and twenty-seven Needs Assessment Checklists (NAC) were completed; 166 of these did not have ethnicity recorded and so were eliminated from further analysis. There were 461 individuals who completed NAC1. Participant demographics can be seen in Table 1.

Study Two. Participants from the original sample were matched in terms of gender, age at injury and level of injury. A total of 42 individuals were matched; there were 14 individuals within each of the three different ethnic groups (white, Asian and black). There were 39 male and 3 female patients. The mean age at injury was 46.6 (s.d. =11.7) and the age range was 26-69. The cause of injury for this subset of the sample was as follows: non-traumatic $=47.6 \%$; road

Table 1 Participant demographics for the sample of patients used in Study One

\begin{tabular}{|c|c|}
\hline \multicolumn{2}{|l|}{ Sex } \\
\hline Male & $342(74.2 \%)$ \\
\hline Female & 119 (25.8\%) \\
\hline \multicolumn{2}{|l|}{ Age } \\
\hline Mean (s.d.) & $47.5(18.2)$ \\
\hline Range & $15-88$ \\
\hline \multicolumn{2}{|l|}{ Ethnicity } \\
\hline White & $393(85.2 \%)$ \\
\hline Black & $35(7.6 \%)$ \\
\hline Asian & $33(7.2 \%)$ \\
\hline \multicolumn{2}{|l|}{ Injury level } \\
\hline C1-C4 (AIS A-C) & $76(18.9 \%)$ \\
\hline C5-C8 (AIS A-C) & 69 (17.0\%) \\
\hline T1-S5 (AIS A-C) & $153(37.6 \%)$ \\
\hline AIS D & $108(26.5 \%)$ \\
\hline Missing & 54 \\
\hline \multicolumn{2}{|l|}{ Cause of injury } \\
\hline Non-traumatic & $33.5 \%$ \\
\hline Falls & $26.7 \%$ \\
\hline RTA & $24.1 \%$ \\
\hline Sport & $11.3 \%$ \\
\hline Assaults & $2.4 \%$ \\
\hline Other & $2.0 \%$ \\
\hline
\end{tabular}

traffic accident $=26.2 \%$; falls $=14.3 \%$; assault $=7.14 \%$; and sport $=$ $4.76 \%$. The categorisation of ASIA Impairment Scale grades was as follows: ASIA Impairment Scale A $=44.7 \%$; ASIA Impairment Scale $\mathrm{B}=10.5 \%$; ASIA Impairment Scale $\mathrm{C}=23.7 \%$; and AIS $\mathrm{D}=21.1 \%$. Participant demographics for each group can be seen in Table 2.

Although there was a slight discrepancy between the groups in terms of cause of injury (traumatic vs non-traumatic), previous research has shown that both patients with traumatic and those with non-traumatic SCI benefit from the same rehabilitation programme and make similar improvements in rehabilitation outcomes from NAC1 to NAC2. ${ }^{18}$

Measurement. The Needs Assessment Checklist ${ }^{19}$ is a comprehensive rehabilitation outcome measure that has demonstrated good reliability and validity. ${ }^{20}$ It was designed specifically for individuals with spinal cord injuries and therefore was the ideal instrument for use in measuring rehabilitation outcomes in the current population. All 10 domains of the NAC are scored using a four-point scale $(0=$ completely dependant; 1 = mostly dependant; $2=$ moderately independent; or 3 =completely independent). The domains consist of physical healthcare, activities of daily living, skin and posture management, bladder management, bowel management, mobility, wheelchair and equipment, community preparation, psychological well-being and discharge coordination. Higher total domain scores therefore indicate higher independence and better rehabilitation outcome. The initial NAC (NAC1) and the second NAC (NAC2) were examined in this study. NAC1 is completed within 2 weeks post mobilisation and NAC2 is completed when the patient is moved into the predischarge ward/6 weeks prior to discharge. Item scores for each NAC domain are totalled, with a percentage calculated indicating 'goal achieved'; these percentage scores were used in the following analyses.

Procedure. Completed NACs were used to (i) compare rehabilitation outcomes broadly within an unmatched sample (Study One), (ii) and to look at the same outcomes but within a matched sample (Study Two). All NACs were administered by trained members of staff who were part of the patient's multi-disciplinary team. Between the two NACs Goal Planning Meetings were held for each patient once every 3 weeks, within which patient-centred goals were discussed and set, giving the patient a structured framework for their rehabilitation.

\section{Data analysis}

Data were analysed using SPSS statistics, version 17.0 (Chicago, IL, USA). Information pertaining to gender, age, level of injury (lesion site and ASIA Impairment Scale) and cause of injury (non-traumatic, road traffic accident, fall, sport or assault) were collected for all individuals. One-way analysis of variances and post hoc tests were conducted to explore differences in rehabilitation outcomes between NAC1 and NAC2 and to ascertain whether differences existed between ethnic groups.

\section{RESULTS}

Study One

NAC1. Outcomes from the first NAC were compared between individuals of White, Asian and Black backgrounds. Significant differences were found within physical healthcare $(\mathrm{F}(2)=3.45$, $P<0.05)$ and psychological well-being $(\mathrm{F}(2)=3.18, P<0.05)$.

The results showed that White individuals had significantly higher scores compared with Black individuals within the domain of physical healthcare $(M=65.03$ vs $58.26 ; t(426)=2.41, P<0.05)$ and within the domain of psychological well-being $(M=62.06$ vs $57.06 ; t(424)=2.00$, 
$P<0.05)$. Higher scores indicated more goals achieved, implying increased independence.

NAC2. The three ethnic groups were then compared using data from the 10 rehabilitation domains of the second NAC. Significant differences were found within physical healthcare $(\mathrm{F}(2)=6.62$, $P<0.01)$, community preparation $(\mathrm{F}(2)=5.96, P<0.01)$ and psychological well-being $(\mathrm{F}(2)=6.95, P<0.01)$.

These results demonstrate that White individuals had significantly higher scores compared with Black individuals $(M=83.20$ vs 76.81$)$ within the domain of physical healthcare $(t(299)=2.32, P<0.05)$. White individuals were also found to score significantly higher than Asian individuals $(M=83.20$ vs 75.35$)$ within physical healthcare $(t(301)=3.00, P<0.01)$.

White individuals were found to have significantly higher scores than Black individuals $(M=70.74$ vs 60.0$)$ within the domain of community preparation $(t(299)=3.25, P<0.01)$.

Table 2 Participant demographics for the matched samples used in Study Two

\begin{tabular}{lccc}
\hline & White & Asian & Black \\
\hline Sex & 13 & 13 & 13 \\
Male & 1 & 1 & 1 \\
Female & & & \\
& & & \\
Age at injury & 46.5 & 46.8 & 46.5 \\
Mean & 11.4 & 12.3 & 12.2 \\
s.d. & & & 7 \\
& 7 & 7 & 6 \\
Level of injury & 6 & 6 & 1 \\
Cervical & 1 & 1 & 8 \\
Thoracic & & & 6 \\
Lumbar & & 8 & \\
Cause of injury & 6 & 6 & \\
Traumatic & 8 & & \\
Non-traumatic & & & \\
\hline
\end{tabular}

White individuals were found to have significantly better scores than Black individuals $(M=80.25$ vs 68.90$)$ within the domain of psychological well-being $(t(299)=3.51, P<0.01)$.

\section{Study Two}

The analysis was then conducted within a sample that was matched for gender, age at injury and level of injury, to examine whether significant differences still existed between ethnic groups in NAC1 and NAC2 scores.

NAC1. A one-way analysis of variance demonstrated significant differences within skin and posture management when looking at NAC1: $\mathrm{F}(2,41)=4.27, P<0.05$. These results indicate that White individuals had significantly higher scores within the domain of skin and posture management compared with Asian individuals $(M=57.07$ vs 35.30$)$.

NAC2. A one-way analysis of variance found significant differences across ethnic groups on NAC2 within the domains of physical healthcare $\quad(\mathrm{F}(2,41)=6.17, \quad P<0.01)$, bowel management $(\mathrm{F}(2,41)=3.30, \quad P<0.05)$ and psychological well-being $(\mathrm{F}$ $(2,41)=3.93, P<0.05)$

These results indicate that White individuals had significantly higher scores within the domain of physical healthcare compared with Asian individuals $(M=87.93$ vs 74.36$)$. In addition, White individuals were also found to score significantly higher than Asian individuals $(M=92.29$ vs 76.14$)$ within the domain of bowel management.

Within the domain of psychological well-being, White individuals were found to score significantly higher than Black individuals $(M=81.43$ vs 68.64$)$

Within-group analysis. Paired sample t-tests indicated that, across the three different ethnic groups, all individuals made significant gains in each of the separate rehabilitation domains from NAC1 to NAC2 and as such showed positive progression during rehabilitation (see Figure 2)

\section{DISCUSSION}

Previous literature has emphasised the need to investigate the relationship between ethnicity and rehabilitation outcomes within SCI. The causal mechanisms behind these differences are still a matter for

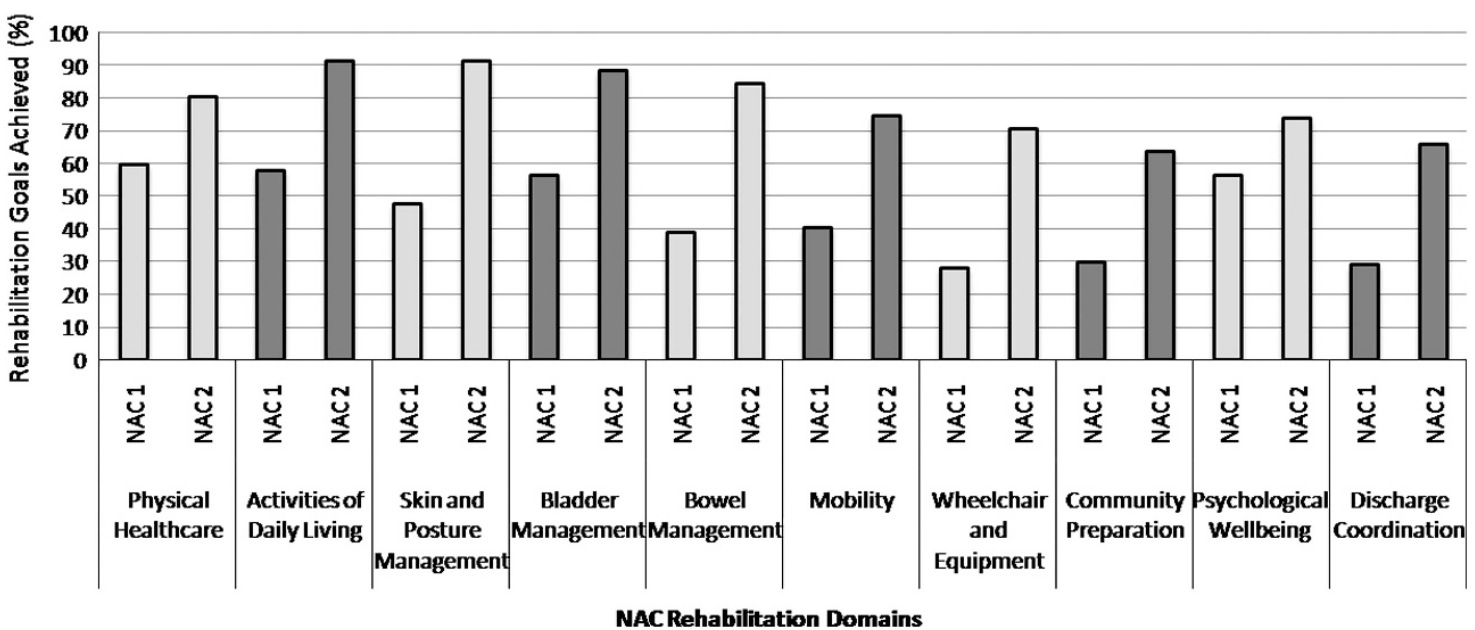

Figure 2 Significant improvement in rehabilitation goals achieved from NAC1 to NAC2 across all 10 rehabilitation domains for all participants in Study Two. 
debate, and there is much disagreement regarding the theory that membership of certain ethnic groups acts more as a proxy than as a predictor variable in explaining outcomes after SCI. ${ }^{16}$ This clinical audit revealed that certain rehabilitation outcomes were significantly different when examining ethnicity in both unmatched and matched samples.

The results from the unmatched data set support previous literature in finding differences within the domains of physical healthcare and psychological well-being on NAC1. In terms of physical healthcare, this is congruent with research that has demonstrated that Black individuals have a higher likelihood of pressure ulcers compared with White individuals. ${ }^{10,12}$ Previous studies also indicate significant differences on account of ethnicity within psychological rehabilitation outcomes, with BME individuals found to score lower when examining 'satisfaction with life' and subjective well-being. ${ }^{14,15}$

When examining NAC2, significant differences were again evident within the psychological well-being and physical healthcare domains between White and Black individuals. In addition to this, White individuals were also shown to demonstrate better rehabilitation outcomes within the domain of community preparation. This is in keeping with the study conducted by Lad et al., ${ }^{9}$ which found there was a longer hospitalisation period for African Americans when compared with Caucasian individuals. Together, these findings suggest that, when examining this unmatched cohort, there is clear evidence to support the notion of rehabilitation outcomes varying significantly between different ethnic groups.

To further examine this finding, the data set was then matched on gender, age at injury and level of injury. In doing so, it was hoped that this would provide a more reliable data set to explore whether significant differences remained in the rehabilitation outcomes previously identified within the unmatched data set.

White individuals were found to score significantly higher than Asian individuals within the physical healthcare domain, which complements the previously found ethnic group differences in the NAC2 unmatched data set. Interestingly, within the matched dataset lower scores were found for Asian individuals, but not for Black individuals, as previously found within the unmatched analysis.

The findings further support there being differences evident in skin management, with Asian patients found to score significantly lower than White individuals. This is in line with the study conducted by Gary et al., ${ }^{8}$ which found that BME patients had a greater likelihood of developing pressure ulcers.

Bowel management was another domain where Asian individuals were found to score significantly lower than White Individuals. This finding is novel in that (i) it was not found within the unmatched data set in this study and (ii) it has not been previously reported in the wider literature and is therefore in need of further study.

White individuals were found to score significantly higher than Black individuals within the psychological well-being domain. This difference was a robust finding within this study, repeated in both the matched and the non-matched data set. Further research is recommended comparing these two groups to identify whether this difference in rehabilitation outcome can be repeated when examining a larger sample, and if so how can we provide psychological interventions to tailor treatment to the needs of Black individuals in rehabilitation. Overall, the results of the matched data set are broadly in line with the findings of the unmatched data, highlighting the differences evident between BME and White individuals on account of predominantly physical healthcare and psychological well-being rehabilitation outcomes.
The methodological strengths of this audit include the following: (i) access to a rich database of information over a lengthy period with outcome measures for key domains of rehabilitation, and (ii) the ability to match these data to reduce the effect of extraneous variables. In addition, the findings are consistent with previous research conducted, and show that within this sample differential rehabilitation outcomes were evident among individuals from different ethnic groups.

\section{Limitations of the current study}

While data matching was used to some extent to minimise the impact of extraneous variables affecting comparisons of the different ethnic groups, this resulted in a relatively small sample size for the matched samples, and as such makes it difficult to draw firm conclusions from the data available. It would be of interest to further investigate whether the results found can be replicated in a larger ethnically diverse matched SCI sample. Furthermore, the ability to match samples on variables such as household income and education level would further minimise the impact of mediating variables and isolate the effect of ethnicity on rehabilitation outcomes. Further research with disadvantaged populations has been identified and recommended as an important need in the coming years, especially in helping to develop targeted population-specific interventions to improve rehabilitation outcomes in areas such as physical healthcare and psychological wellbeing. $^{21}$

\section{CONCLUSIONS}

This is the first known study within the UK, to our knowledge, to objectively compare rehabilitation outcomes in SCI as a consequence of ethnicity. Previous research has been conducted predominantly within the US, where, due to marked differences in healthcare provision, there are important differences affecting rehabilitation outcome overall.

Within the wider literature, there are clear discrepancies in rehabilitation outcomes when comparing individuals from different ethnic groups, with the casual mechanisms behind this yet to reach a consensus. Differential outcomes were evident in the current study within the rehabilitation domains of physical healthcare and psychological well-being. Further research is needed to develop a better understanding of whether these differences can be replicated within a larger, more representative, sample, and if so how can rehabilitation be tailored to best help these currently disadvantaged individuals.

\section{CONFLICT OF INTEREST}

The authors declare no conflict of interest.

\section{ACKNOWLEDGEMENTS}

We thank all patients and staff at The National Spinal Injuries Centre, Stoke Mandeville Hospital, who participated in this clinical audit.

1 Bhopal R. Race, Ethnicity and Health in Multicultural Societies. Foundations for Better Epidemiology, Public Health and Health Care. Oxford. OUP: Oxford, UK, 2007.

2 Aspinall PJ, Jacobson B. Ethnic Disparities in Health and Health Care-A Focused Review of the Evidence and Selected Examples of Good Practice. London Health Observatory/Department of Health, 2004.

3 Krause JS, Broderick L. Outcomes after spinal cord injury: comparisons as a function of gender and race and ethnicity. Arch Phys Med Rehabil 2004; 95 355-36.

4 Marmot M. Fair Society, Healthy Lives: the Marmot Review: Strategic Review of Health Inequalities in England post-2010. Marmot Review: London, UK, 2010. 
5 Nazroo JY. The structuring of ethnic inequalities in health: economic position, racial discrimination and racism. Am J Public Health 2003; 93: 277-284.

6 Krause JS, Broderick LE, Saladin LS, Broyles J. Racial disparities in health outcomes after spinal cord injury: mediating effects of education and income. J Spinal Cord Med 2006; 29: 17-25.

7 Fyffe DC, Botticello AL, Myaskovsky L. Vulnerable Groups Living with Spinal Cord Injury. Top Spinal Cord Inj Rehabil 2011; 17: 1-9.

8 Gary KW, Nicholls E, Shamburger A, Stevens LF, Arango-Lasprilla JC. Do racial and ethnic minority patients fare worse after SCI? A critical review of the literature. NeuroRehabilitation 2011; 29: 275-293.

9 Lad SP, Umeano OA, Karikari IO, Somasundaram A, Bagley CA, Gottfried ON et al. Racial disparities in outcomes after spinal cord injury. J Neurotrauma 2013; 30: 492-497.

10 Waters RL, Adkins RH. Firearm versus motor vehicle related spinal cord injury: preinjury factors, injury characteristics, and initial outcome comparisons among ethnically diverse groups. Arch Phys Med Rehabil 1997; 78: 150-155.

11 Waters RL, Adkins RH, Sie I, Cressy J. Post rehabilitation outcomes after spinal cord injury caused by firearms and motor vehicle crash among ethnically diverse groups. Arch Phys Med Rehabil 1998; 79: 1237-1243.

12 Saladin LS, Krause JS. Pressure ulcer prevalence and barriers to treatment after spinal cord injury: comparisons of four groups based on race-ethnicity. NeuroRehabilitation 2009; 24: 57-66.
13 Fyffe D, Deutsch A, Botticello A, Kirshblum S, Ottenbacher K. Racial and ethnic disparities in functioning at discharge and follow-up among patients with motor complete spinal cord injury. Arch Phys Med Rehabil 2014; 95: 2140-2151.

14 Arango-Lasprilla JC, Ketchum JM, Gary K, Hart T, Corrigan J, Forster L et al. Race/ ethnicity differences in satisfaction with life among people with traumatic brain injury. Neuro Rehabilitation 2009; 24: 5-14.

15 Krause JS, Saladin LS, Adkins RH. Disparities in subjective well-being, participation, and health after spinal cord injury: a 6-year longitudinal study. Neuro Rehabilitation 2009; 24: 47-56.

16 Putze JD, Hickens BL, Richards JS. Race: predictor versus proxy variable? Outcomes after spinal cord injury. Arch Phys Med Rehabil 2002; 83: 1603-1611.

17 Meade MA, Lewis A, Jackson MN, Hess DW. Race employment, and spinal cord injury. Arch Phys Med Rehabil 2004; 85: 1782-1792.

18 Kennedy P, Chessell Z J. Traumatic versus non-traumatic spinal cord injuries: are there differential rehabilitation outcomes? Spinal Cord 2013; 51: 579-583.

19 Kennedy P, Hamilton LR. The needs assessment checklist: a clinical approach to measuring outcome. Spinal Cord 1999; 37: 136-139.

20 Berry C, Kennedy P. A psychometric analysis of the Needs Assessment Checklist (NAC). Spinal Cord 2003; 41: 490-501.

21 Pyatak, Blanche El, Garber SL, Diaz J, Blanchard J, Florindez L et al. Conducting intervention research among underserved populations: lessons learned and recommendations for researchers. Arch Phys Med Rehabil 2013; 94: 1190-1198. 